\title{
Chemotherapy for intracranial ependymoma in adults
}

\author{
Dorothee Gramatzki ${ }^{*}$, Patrick Roth ${ }^{1}$, Jörg Felsberg ${ }^{2,3}$, Silvia Hofer ${ }^{4}$, Elisabeth J. Rushing ${ }^{5}$, Bettina Hentschel ${ }^{6}$, \\ Manfred Westphal ${ }^{7}$, Dietmar Krex ${ }^{8}$, Matthias Simon ${ }^{9}$, Oliver Schnell ${ }^{10}$, Wolfgang Wick ${ }^{11,12}$, \\ Guido Reifenberger ${ }^{12,3}$ and Michael Weller ${ }^{1,12}$
}

\begin{abstract}
Background: Ependymal tumors in adults are rare, accounting for less than $4 \%$ of primary tumors of the central nervous system in this age group. The low prevalence of intracranial ependymoma in adults limits the ability to perform clinical trials. Therefore, treatment decisions are based on small, mostly retrospective studies and the role of chemotherapy has remained unclear.

Methods: We performed a retrospective study on 17 adult patients diagnosed with intracranial World Health Organisation grade II or III ependymoma, who were treated with chemotherapy at any time during the disease course. Benefit from chemotherapy was estimated by applying Macdonald criteria. Progression-free (PFS) and overall survival (OS) were calculated from start of chemotherapy, using the Kaplan-Meier method.

Results: Eleven patients had supratentorial and 6 infratentorial tumors. Ten patients were treated with temozolomide (TMZ), 3 with procarbazine/lomustine/vincristine (PCV), 3 with platinum-based chemotherapy and 1 patient received epirubicin/ifosfamide. Response rates were as follows: TMZ 8/10 stable disease; PCV 3/3 stable disease; platinum-based chemotherapy $1 / 3$ partial response; epirubicin/ifosfamide 1/1 complete response. PFS rates at 6, 12 and 24 months were 52.9, 35.3 and $23.5 \%$. OS rates at 6, 12 and 24 months were 82.4, 82.4 and $70.1 \%$. There was no indication for a favourable prognostic role of $\mathrm{O}^{6}$-methylguanyl-DNA-methyltransferase (MGMT) promoter methylation which was detected in 3/12 investigated tumors.

Conclusions: Survival outcomes in response to chemotherapy in adult intracranial ependymoma patients vary substantially, but individual patients may respond to any kind of chemotherapy. There were too few patients to compare survival data between chemotherapeutic subgroups.
\end{abstract}

Keywords: Adults, Chemotherapy, Intracranial ependymoma, Overall survival, Progression-free survival

\section{Background}

Ependymomas are a histologically, biologically and clinically heterogenous group of glial tumors that show histological features of ependymal differentiation and preferentially are located in the cerebral ventricles or the spinal cord. In total, ependymomas account for $6.8 \%$ of all gliomas, with the relative frequency being higher in children compared to adults [1]. In adults, ependymomas are less than $4 \%$ of primary central nervous system tumors [2] and are found more often in spinal (46\%)

\footnotetext{
* Correspondence: dorothee.gramatzki@usz.ch

${ }^{1}$ Department of Neurology, University Hospital Zurich, Frauenklinikstrasse 26, 8091 Zurich, Switzerland

Full list of author information is available at the end of the article
}

than infra- (35\%) or supratentorial (19\%) locations [3]. These tumors are classified by the World Health Organisation (WHO) into 3 grades [4]. The prognostic relevance of the histopathological distinction between WHO grade II versus WHO grade III ependymomas has remained controversial $[2,5]$. In comparison to $\mathrm{WHO}$ grade II ependymomas, WHO grade III ependymomas are associated with increased risk of treatment failure [3].

The low prevalence of intracranial ependymoma in adults limits opportunities to perform large clinical trials. Thus, treatment recommendations are based on small, mostly retrospective studies. Surgical resection is the most important therapeutic intervention for intracranial ependymomas [2]. Extent of resection has been 
associated with increased progression-free survival (PFS) and overall survival (OS) in most series [3, 6-8]. Adjuvant radiotherapy (RT) is recommended for patients diagnosed with anaplastic (WHO grade III) ependymoma $[2,9]$, whereas the role of RT in patients with WHO grade II ependymoma is discussed controversially. While resection followed by irradiation as first-line therapy is considered for posterior fossa ependymoma [10], RT for supratentorial ependymoma is commonly used only when surgical resection has been incomplete $[2,3,11]$. The role of chemotherapy (CT) for intracranial ependymoma in adults remains unclear. A variety of chemotherapeutic drugs has been investigated in the past, mostly in retrospective studies or case reports. Selected case reports demonstrate response to temozolomide (TMZ) in recurrent WHO grade III ependymoma [12, 13] or recurrent WHO grade II and III ependymomas [14]. Activity of TMZ was also described in a retrospective study, analyzing recurrent WHO grade II ependymomas, refractory for platinum-based chemotherapy [15], as well as in a prospective phase II study in ependymoma patients treated with TMZ and lapatinib at tumor recurrence [16]. Brandes and colleagues compared patients with recurrent WHO grade II ependymomas treated with cisplatin-based CT or treated with another kind of CT, demonstrating no significant differences regarding PFS or OS [17]. Here we performed a retrospective analysis of outcome data in 17 adult patients diagnosed with intracranial WHO grade II or III ependymoma and treated with chemotherapy at any time during their disease course.

\section{Methods}

\section{Patients and tumors}

In accordance with approval from the appropriate Institutional Review Boards, the surgical specimens and clinical records were retrieved from 17 patients treated at the University Hospital Zurich, Zurich, Switzerland, or at one of the eight University Hospitals in Germany participating in the German Glioma Network (GGN) (http://www.gliomnetzwerk.de). All patients gave written informed consent according to the research proposals approved by the Institutional Review Boards of the participating institutions (University of Zurich, Switzerland; Universities of Bochum, Bonn, Dresden, Düsseldorf, Hamburg, Heidelberg, Munich and Tübingen, all Germany). All tumors were classified and graded according to the WHO classification of tumors of the central nervous system [4]. The $O^{6}$-methylguanyl-DNA-methyltransferase (MGMT) promoter methylation status was determined by methylation-specific polymerase chain reaction in 12 tumors [18]. Epidemiological and treatment data were taken from patient health records. Radiological response rates to chemotherapy were documented using
Macdonald criteria [19] as foreseen in the German Glioma Network study protocol.

\section{Statistics}

PFS and OS curves were estimated by the KaplanMeier method. PFS was calculated from the date of first chemotherapy to the date of progression. OS was measured from the date of first chemotherapy to the date of death. Patients without confirmed death were censored for OS at the last follow-up visit. Patients without documented progression were censored at the last follow-up visit for PFS. Survival-related analyses were calculated with the log-rank test. A cox proportional hazard model was used for univariate analysis, to test the association of clinical predictors with survival outcomes from start of chemotherapy. All statistical tests were two-tailed, and a $p$ value of 0.05 was set as statistically significant. All statistical analyses were performed using Prism 6 (GraphPad Software) or Statistics 22 (SPSS software).

\section{Results}

\section{Patient characteristics}

Table 1 summarizes the principle patient characteristics: 17 patients initially diagnosed with intracranial ependymoma WHO grade II or III were studied. Median age at diagnosis was 28 years (range 18-56 years, mean age 33 years, $95 \%$ confidence interval (CI) 27-40); 14 patients were men $(82.4 \%) ; 11$ patients had supratentorial $(64.7 \%)$ and 6 patients had infratentorial (35.3\%) tumors. Most patients (58.8 \%) had a Karnofsky Performance Score (KPS) of $90-100 \%$ at the time of initial diagnosis. At time of first chemotherapy, most patients (52.9\%) had a KPS of 70-80 \%. Histology at time of first surgical intervention revealed ependymoma WHO grade II in 4 patients and anaplastic ependymoma WHO grade III in 13 patients. Nine patients were treated with RT alone $(52.9 \%)$ as first-line therapy post-surgery, 2 patients with anaplastic ependymoma received radiotherapy plus concomitant and maintenance temozolomide chemotherapy (TMZ/RT $\rightarrow$ TMZ) [20] at this timepoint, while 6 patients did not receive upfront treatment. At recurrence, 11 patients (64.7\%) underwent another surgical tumor resection and 6 patients (35.3\%) received CT treatment at this time. The remaining 9 patients received the first $\mathrm{CT}$ treatment at subsequent recurrences. In all cases tumor recurrence was local when $\mathrm{CT}$ was started. Three patients (patients 7, 8 and 14) experienced spinal drop metastases later on in the course of disease. Eight patients (47.1 \%) were alive at a median follow-up period of 87 months. Median follow-up, defined from start of first chemotherapy, was 39 months for 
Table 1 Summary of patient characteristics

\begin{tabular}{ll}
\hline & $\mathrm{n}=17$ patients \\
\hline Age (years) & \\
Median & 28 \\
Range & $18-56$
\end{tabular}

Age classes, n (\%)

$\leq 20$ years

$2(11.8)$

21-30 years

$8(47.1)$

$31-40$ years

41-50 years

5 (29.4)

$>50$ years

$2(11.8)$

Gender

Female

$3(17.6)$

Male

$14(82.4)$

KPS (pre-operative), n (\%)

90-100

$10(58.8)$

70-80

$<70$

No data

$6(35.3)$

0

$1(5.9)$

KPS (start first CT), n (\%)

90-100

$3(17.6)$

70-80

$13(76.5)$

$<70$

0

No data

$1(5.9)$

Tumor localizationc

Supratentorial

$11(64.7)$

Infratentorial

$6(35.3)$

Extent of first resection, $\mathrm{n}(\%)$

Gross total resection

Subtotal resection

5 (29.4)

Partial resection

$5(29.4)$

Biopsy

$2(11.8)$

No data

$1(5.9)$

Histology (initial)

Ependymoma WHO grade II

Anaplastic ependymoma WHO grade III

$13(76.5)$

Histology (start first CT)

Ependymoma WHO grade II

$1(5.9)$

Anaplastic ependymoma

$14(82.4)$

Sarcoma/Gliosarcoma

$2(11.8)$

MGMT promoter methylation status, $\mathrm{n}(\%)$

Unmethylated

$9(52.9)$

Methylated

$3(17.6)$

No data

$5(29.1)$

First-line therapies beyond surgery, n (\%)

RT alone
Table 1 Summary of patient characteristics (Continued)

\begin{tabular}{ll}
\hline TMZ/RT $\rightarrow$ TMZ & $2(11.8)$ \\
No therapy & $6(35.3)$ \\
First salvage therapy, $\mathrm{n}(\%)$ & \\
Re-resection & \\
alone & $4(23.5)$ \\
plus RT alone & $2(11.8)$ \\
plus RT plus TMZ & $2(11.8)$ \\
plus Bevacizumab & $1(5.9)$ \\
plus CT & $2(11.8)$ \\
CT alone & \\
RT alone & $4(23.5)$ \\
Bevacizumab alone & $1(5.9)$ \\
\end{tabular}

First CT, n (\%)

TMZ

$10(58.8)$

Procarbazine plus Lomustine plus Vincristine 3 (17.6)

Epirubicin plus Ifosfamide 1 (5.9)

Carboplatin plus Etoposide 2 (11.8)

Carboplatin plus Etoposide plus Vincristine $\quad 1$ (5.9)

Number of surgical interventions

1

$5(29.4)$

2

$3(17.6)$

$>3$

$9(52.9)$

Survival (from first $C T$ ) (all patients $n=17$ )

Median follow-up (months)

39

Median PFS (months) (95\% Cl) (events)

$10(3.4-16.6)(15)$

Median OS (months) (95\% Cl) (events)

$41(31.6-50.4)(9)$

Survival (from first CT)

$\left(n=15\right.$, patients 5 and 9 excluded $\left.^{d}\right)$

Median follow-up (months) 39

Median PFS (months) (95\% Cl) (events) 6 (1.5-10.5) (14)

Median OS (months) (95\% Cl) (events)

$41(30.0-52)(8)$

Cl confidence interval, CT chemotherapy, KPS Karnofsky Performance Score, $M G M T O^{6}$-methylguanyl-DNA-methyltransferase, n.a. not applicable, OS overall survival, $P C V$ procarbazine/lomustine/vincristine, PFS progression-free survival, $R T$ radiotherapy, $T M Z$ temozolomide

a , 1 PCV, 1 TMZ; ${ }^{\text {b }, ~} 1$ PCV, 2 TMZ, 1 carboplatin plus etoposide; ${ }^{\text {c }}$, tumor localization was the same at date of diagnosis and start of $\mathrm{CT} ;{ }^{d}$, patients 5 and 9 were diagnosed with sarcoma or gliosarcoma at recurrence

the whole patient group. Individual patient profiles are summarized in Table 2.

At time of recurrence 2 patients initially diagnosed with anaplastic ependymoma were diagnosed with sarcoma (patient 5) or gliosarcoma (patient 9) (Table 2). Both patients had been previously treated with RT. At recurrence patient 5 was treated with 7 cycles of epirubicin/ifosfamide and demonstrated a complete response $(\mathrm{CR})$ with no evidence of recurrence during follow-up of 8.8 years. Patient 9 was treated with 9 cycles of TMZ, best response was 
Table 2 Individual patient characteristics ${ }^{c}$

\begin{tabular}{|c|c|c|c|c|c|c|c|c|c|c|c|c|c|c|c|}
\hline No. & $\begin{array}{l}\text { Initial } \\
\text { histology }\end{array}$ & $\begin{array}{l}\text { WHO } \\
\text { grade }\end{array}$ & $\begin{array}{l}\text { MGMT } \\
\text { status }\end{array}$ & Localization & $\begin{array}{l}\text { Extent of } \\
\text { resection }\end{array}$ & $\begin{array}{l}\text { First-line } \\
\text { therapy }\end{array}$ & $\begin{array}{l}\text { First salvage } \\
\text { therapy }\end{array}$ & $\begin{array}{l}\text { Surgical } \\
\text { interventions ( } n \text { ) }\end{array}$ & $\begin{array}{l}\text { Histology at } \\
\text { start of CT }\end{array}$ & First CT & $\begin{array}{l}\text { Duration of } \\
\text { first } C T^{a}\end{array}$ & $\begin{array}{l}\text { Best } \\
\text { response }\end{array}$ & $\mathrm{DBR}^{\mathrm{a}}$ & $\mathrm{PFS}^{\mathrm{a}}$ & $\mathrm{OS}^{\mathrm{a}}$ \\
\hline \multirow[t]{2}{*}{$\overline{P 1}$} & $\mathrm{E}$ & $\|$ & unmethyl. & infratentorial & subtotal & - & RT & 3 & $A E$ & TMZ & 11 & SD & 11 & 17 & $68^{b}$ \\
\hline & & & & IV ventricle & & & & & & & & & & & \\
\hline \multirow[t]{2}{*}{ P2 } & E & $\|$ & unmethyl. & supratentorial & partial & RT & re-resection RT & $>3$ & AE & PCV & 18 & SD & 4 & 21 & 41 \\
\hline & & & & parietal & & & & & & & & & & & \\
\hline \multirow[t]{4}{*}{ P3 } & E & $\|$ & unmethyl. & infratentorial & partial & - & re-resection & 2 & $\mathrm{AE}$ & $\mathrm{TMZ} / \mathrm{RT} \rightarrow \mathrm{TMZ}$ & 8 & SD & 29 & $32^{b}$ & $32^{b}$ \\
\hline & & & & cerebellar & & & $\mathrm{TMZ} / \mathrm{RT} \rightarrow \mathrm{TMZ}$ & & & & & & & & \\
\hline & & & & brainstem & & & & & & & & & & & \\
\hline & & & & IV ventricle & & & & & & & & & & & \\
\hline \multirow[t]{2}{*}{ P4 } & E & $\|$ & unmethyl. & supratentorial & partial & - & re-resection & 3 & $\mathrm{E}$ & $\mathrm{TMZ}$ & 6 & SD & 2 & 5 & 5 \\
\hline & & & & III ventricle & & & & & & & & & & & \\
\hline \multirow[t]{4}{*}{ P5 } & $\mathrm{AE}$ & III & n.d. & supratentorial & gross total & RT & re-resection & 2 & sarcoma & epirubicin & 5 & $C R$ & 106 & $131^{b}$ & $131^{b}$ \\
\hline & & & & parietal & & & & & & ifosfamide & & & & & \\
\hline & & & & temporal & & & & & & & & & & & \\
\hline & & & & occipital & & & & & & & & & & & \\
\hline \multirow[t]{2}{*}{ P6 } & $\mathrm{AE}$ & III & unmethyl. & infratentorial & subtotal & RT & carboplatin & 1 & $A E$ & carboplatin & 5 & $\mathrm{PR}$ & 7 & 11 & $16^{\mathrm{b}}$ \\
\hline & & & & IV ventricle & & & etoposide & & & etoposide & & & & & \\
\hline \multirow[t]{4}{*}{ P7 } & $\mathrm{AE}$ & III & n.d. & supratentorial & partial & - & re-resection & $>3$ & $\mathrm{AE}$ & carboplatin & 1 & PD & - & 2 & $44^{b}$ \\
\hline & & & & parietal & & & & & & etoposide & & & & & \\
\hline & & & & & & & & & & vincristine & & & & & \\
\hline & & & & & & & & & & $\begin{array}{l}\text { cyclo- } \\
\text { phosphamide }\end{array}$ & & & & & \\
\hline \multirow[t]{2}{*}{ P8 } & $\mathrm{AE}$ & $\|$ & unmethyl. & infratentorial & gross & - & re-resection & $>3$ & AE & $\mathrm{TMZ}$ & 17 & SD & 21 & 25 & $80^{b}$ \\
\hline & & & & IV ventricle & total & & RT & & & & & & & & \\
\hline \multirow[t]{3}{*}{ P9 } & $\mathrm{AE}$ & III & n.d. & supratentorial & biopsy & RT & re-resection & 3 & gliosarcoma & $\mathrm{TMZ}$ & 9 & SD & 7 & 10 & 23 \\
\hline & & & & frontal & & & TMZ & & & & & & & & \\
\hline & & & & temporal & & & & & & & & & & & \\
\hline \multirow[t]{2}{*}{ P10 } & $\mathrm{AE}$ & III & n.d. & infratentorial & gross & RT & TMZ & 1 & $A E$ & TMZ & 5 & SD & 31 & 33 & $101^{b}$ \\
\hline & & & & cerebellar & total & & & & & & & & & & \\
\hline \multirow[t]{2}{*}{ P11 } & $\mathrm{AE}$ & III & unmethyl. & supratentorial & subtotal & RT & TMZ & 1 & $A E$ & TMZ & 9 & SD & 9 & 10 & 13 \\
\hline & & & & III ventricle & & & & & & & & & & & \\
\hline \multirow[t]{2}{*}{ P12 } & $\mathrm{AE}$ & III & n.d. & supratentorial & subtotal & $\mathrm{TMZ} / \mathrm{RT} \rightarrow$ & re-resection & $>3$ & $A E$ & $\mathrm{TMZ} / \mathrm{RT} \rightarrow \mathrm{TMZ}$ & 5 & SD & 4 & 5 & 48 \\
\hline & & & & parietal & & & bevacizumab & & & & & & & & \\
\hline
\end{tabular}


Table 2 Individual patient characteristics ${ }^{c}$ (Continued)

\begin{tabular}{|c|c|c|c|c|c|c|c|c|c|c|c|c|c|c|c|}
\hline P13 & $\mathrm{AE}$ & III & unmethyl. & $\begin{array}{l}\text { supratentorial } \\
\text { parietal }\end{array}$ & gross total & RT & $\begin{array}{l}\text { re-resection } \\
\text { PCV }\end{array}$ & 3 & $\mathrm{AE}$ & PCV & 8 & SD & 1 & 5 & 40 \\
\hline P14 & $\mathrm{AE}$ & III & unmethyl. & $\begin{array}{l}\text { supratentorial } \\
\text { temporal } \\
\text { III ventricle }\end{array}$ & biopsy & - & $\begin{array}{l}\text { re-resection } \\
\text { RT } \\
\text { TMZ }\end{array}$ & 2 & $A E$ & $\mathrm{TMZ}$ & 1 & PD & - & 2 & 2 \\
\hline P15 & $\mathrm{AE}$ & III & methyl. & $\begin{array}{l}\text { infratentorial } \\
\text { cerebellar } \\
\text { brainstem } \\
\text { IV ventricle }\end{array}$ & partial & RT & re-resection & 3 & $\mathrm{AE}$ & $\begin{array}{l}\text { carboplatin } \\
\text { etoposide }\end{array}$ & n.d. & PD & - & 4 & $29^{\mathrm{b}}$ \\
\hline P16 & $\mathrm{AE}$ & III & methyl. & $\begin{array}{l}\text { supratentorial } \\
\text { temporal }\end{array}$ & n.d. & RT & PCV & 1 & $\mathrm{AE}$ & PCV & 3 & SD & 2 & 6 & 39 \\
\hline P17 & $\mathrm{AE}$ & III & methyl. & $\begin{array}{l}\text { supratentorial } \\
\text { parietal }\end{array}$ & subtotal & $\begin{array}{l}\text { TMZ/RT } \rightarrow \\
\text { TMZ }\end{array}$ & bevacizumab & 1 & $A E$ & $\mathrm{TMZ} / \mathrm{RT} \rightarrow \mathrm{TMZ}$ & 1 & PD & - & 1 & 6 \\
\hline
\end{tabular}

AE anaplastic ependymoma, $C R$ complete response, DBR duration best response, $E$ ependymoma WHO grade Il; unmethyl., unmethylated, MGMT status $O^{6}$-methylguanyl-DNA-methyltransferase promoter methylation status, n.d. no data, no. number, $O S$ overall survival, $P$ patient, $P C V$ procarbazine/lomustine/vincristine, $P D$ progressive disease, $P F S$ progression-free survival, $P R$ partial response, $R T$ radiotherapy, $S D$ stable disease, $T M Z$ temozolomide

${ }^{a}$ in months; ${ }^{b}$ indicates patients who did not demonstrate progressive disease decease or who were not deceased, ${ }^{c}$ median age was 28 years (range $18-56$ ), 3 female and 14 male patients were included 
stable disease (SD) with PFS of 10 months and OS of 23 months.

\section{Benefit from chemotherapy}

At time of first chemotherapeutic treatment, 10 patients were treated with TMZ (58.8 \%), 3 patients with procarbazine/lomustine/vincristine (PCV) (17.6\%), 3 patients with platinum-based CT (carboplatin/etoposide (11.8\%) or carboplatin/etoposide/vincristine (5.9\%)) and 1 patient with epirubicin/ifosfamide (5.9\%) (Table 1). Two patients received $\mathrm{CT}$ as first line therapy, 8 patients at time of first recurrence and 7 patients at time of second recurrence (Table 2). Median PFS after first chemotherapeutic treatment was 10 months (95\% CI 3.4-16.6) for all patients pooled, as it was for the group of patients treated with TMZ (95\% CI 2.4-17.6); it was 4 (95\% CI 0.8-7.2) months for patients treated with platinum-based CT and 6 months (95\% CI 4.4-7.6) for patients treated with PCV. PFS for the patient treated with epirubicin/ifosfamide was not reached during a follow-up period of 131 months. Median OS from start of chemotherapy was 41 months $(95 \%$ CI 31.6-50.4) for all patients pooled, 23 months (95\% CI 0-71.2) for patients treated with TMZ and 40 months (95\% CI 38.4-41.6) for patients treated with PCV. OS in the group of patients treated with platinum-based CT was not reached during the follow-up period. Since two patients (patients 5 and 9), treated with epirubicin/ifosfamide or TMZ, were not diagnosed with classical ependymoma at time of start of chemotherapy, survival data were also assessed for the remaining 15 patients: median PFS after first chemotherapeutic treatment was 6 months (95 \% CI 1.5-10.5) for all patients pooled $(n=15)$, and 10 months (95 \% CI 0.0-24.6) for patients treated with TMZ; median OS after first chemotherapeutic treatment was 41 months (95\% CI 30.0-52) for all patients pooled $(n=15)$, and 48 months $(95 \%$ CI 0.0-133.4) for patients treated with TMZ. In the group of patients treated with TMZ, 8 patients showed SD and 2 patients showed progressive disease (PD). In the group of patients treated with PCV, all 3 patients had $\mathrm{SD}$ and among the 3 patients treated with platinum-based CT, 1 patient demonstrated partial response (PR) and 2 patients $\mathrm{PD}$. The patient who received epirubicin/ifosfamide (patient 5) demonstrated CR (Table 2). PFS and OS for all patients are shown in Fig. 1. PFS rates at 6, 12 and 24 months were 52.9, 35.3 and $23.5 \%$ for all 17 patients included in this study, and 46.7, 33.3 and $33.3 \%$ when the two patients diagnosed with sarcoma or gliosarcoma at time of recurrence were excluded. OS rates at 6,12 and 24 months were $82.4,82.4$ and $70.1 \%$ for all patients, and $80.0,80.0$ and $73.3 \%$ for the

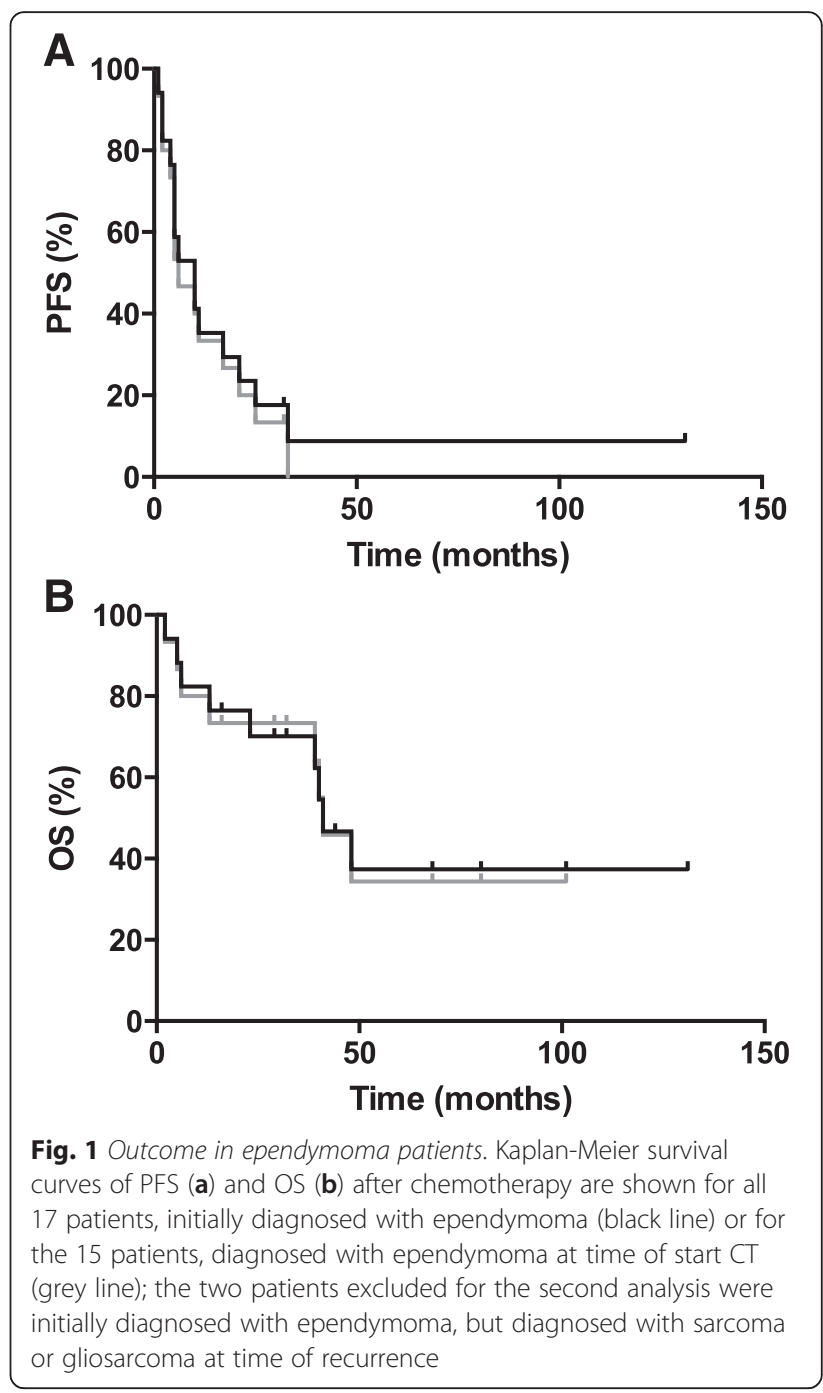

reduced patient cohort $(n=15)$. Group sizes were too small for formal comparisons, but not obvious signal of activity of a particular regimen became apparent.

\section{MGMT promoter methylation and survival in intracranial ependymoma}

The MGMT promoter methylation status of the tumor was available in 12 patients (Table 2): 3 patients had MGMT promoter methylated tumors, whereas 9 patients did not. Patients with MGMT promoter methylated tumors were treated with TMZ, platinum-based CT or PCV. Patients without MGMT promoter methylation were treated with TMZ (6 patients), platinum-based CT (1 patient) or PCV (2 patients). Median PFS was 4 months for patients with $M G M T$ promoter methylated tumors and 11 months for patients with MGMT promoter unmethylated tumors. Median OS was 39 months for patients with $M G M T$ promoter methylated tumors versus 40 months for patients with MGMT promoter 
unmethylated tumors. Group sizes were too small for formal comparisons between patients stratified according to the MGMT promoter methylation status.

\section{Association of age, tumor localization, gender, KPS and MGMT promoter methylation status with survival}

Patients were divided into two groups, defined by age, tumor localization, gender, KPS and MGMT promoter methylation status, and a log-rank survival analysis was performed. This analysis revealed supratentorial tumors to be associated with inferior survival (Table 3, Additional file 1: Table S1). Univariate analysis using the cox proportional hazard model from start of first CT treatment was performed to identify factors associated with overall survival. The results of these analyses are summarized in Table 3 for all 17 patients initially diagnosed with ependymoma, and in Additional file 1: Table S1 for the remaining patient cohort when the two patients diagnosed with sarcoma or glioblastoma at recurrence were excluded. Age, tumor localization, gender, KPS and the MGMT promoter methylation status were not identified as risk factors for death in both patient cohorts (Table 3, Additional file 1: Table S1).

\section{Discussion}

Intracranial ependymoma is a rare disease in adults [2]. While the role of surgical resection and RT for tumor control is undisputed [2, 3, 6-9, 11], little is known about the significance of CT. Here we retrospectively analyzed 17 adult patients with intracranial $\mathrm{WHO}$ grade II or grade III ependymomas to investigate the impact of chemotherapy on the disease course.

Most patients $(n=10)$ received TMZ as their first chemotherapeutic treatment (Table 1). Two of these patients received a combined treatment of irradiation and TMZ (TMZ/RT $\rightarrow$ TMZ) [20] at diagnosis. Median PFS for these two patients was low with 3 months as it was for median OS with 27 months. The other 8 patients were treated with TMZ at recurrence and the best response was SD. Thus, TMZ demonstrated some activity in intracranial ependymoma patients, especially at time of recurrence. Of note, there is one prospective study presented at the American Society of Clinical Oncology (ASCO) in 2005, which included patients with recurrent WHO grade II and III ependymomas. Activity of TMZ treatment in these patients was as follows (best response: median PFS): CR $(n=2)$ : 9-48 months; PR $(n=$ 3): 4-15 months; SD $(n=5): 7-44$ months [14] (Table 4). Less optimal results were reported by Chamberlain and Johnston for TMZ treatment of 25 patients with recurrent WHO grade II ependymomas, refractory to platinum-based chemotherapy, who demonstrated a median PFS of 3 months, and best responses of PR in 1 patients and SD in 9 patients with a median OS of 3 months [15] (Table 4). Moreover, results of a prospective trial, including 24 patients diagnosed with WHO grade II ependymoma and 18 patients diagnosed with anaplastic ependymoma, treated with TMZ plus

Table 3 Association of age, tumor localization, gender, KPS and MGMT promoter methylation status with survival (all patients, $n=17$ )

\begin{tabular}{|c|c|c|c|c|c|}
\hline Variable & $\begin{array}{l}\text { Number of patients } \\
\text { (events) }\end{array}$ & $\begin{array}{l}\text { Median OS (months) } \\
(95 \% \mathrm{Cl})\end{array}$ & $\begin{array}{l}\text { p value } \\
\text { (log-rank) }\end{array}$ & $\begin{array}{l}p \text { value } \\
\text { (cox regression) }\end{array}$ & $\begin{array}{l}\text { Hazard Ratio } \\
(95 \% \text { Cl) }\end{array}$ \\
\hline \multicolumn{6}{|l|}{$\overline{\mathrm{Age}^{\mathrm{a}}}$} \\
\hline$<40$ years & $9(5)$ & $48(28.5-67.5)$ & & & 1 \\
\hline$\geq 40$ years & $8(4)$ & $41(13.8-68.14)$ & 0.587 & 0.589 & $1.45(0.37-5.64)$ \\
\hline \multicolumn{6}{|l|}{ Tumor localization ${ }^{a}$} \\
\hline Infratentorial & $6(0)$ & undefined & & & 1 \\
\hline Supratentorial & $11(9)$ & $39(9.87-68.13)$ & $0.011\left(^{*}\right)$ & 0.163 & $50.8(0.20-12662.82)$ \\
\hline \multicolumn{6}{|l|}{ Gender } \\
\hline Female & $3(2)$ & $41(0-97.01)$ & & & 1 \\
\hline Male & $14(7)$ & $48(35.84-60.16)$ & 0.888 & 0.888 & $0.89(0.18-4.34)$ \\
\hline \multicolumn{6}{|l|}{$\mathrm{KPS}^{\mathrm{a}}$} \\
\hline $100-80$ & $11(6)$ & $48(14.47-81.54)$ & & & 1 \\
\hline$<80$ & $5(3)$ & $41(11.96-70.04)$ & 0.872 & 0.872 & $1.12(0.28-4.52)$ \\
\hline \multicolumn{6}{|c|}{ MGMT promoter methylation } \\
\hline not methylated & $9(5)$ & $40(9.3-70.7)$ & & & 1 \\
\hline methylated & $3(2)$ & 39 (undefined) & 0.433 & 0.443 & $2.02(0.34-12.2)$ \\
\hline
\end{tabular}

Cl confidence interval, KPS Karnofsky Performance Score, WHO World Health Organisation

${ }^{*}, p<0.05$

${ }^{a}$, variables were determined at start of chemotherapy 
Table 4 Review of the literature: chemotherapeutic treatment regimens in adult intracranial ependymoma

\begin{tabular}{|c|c|c|c|c|c|c|c|}
\hline $\begin{array}{l}\text { First author, } \\
\text { year [Ref] }\end{array}$ & Trial design & $\begin{array}{l}\text { Patient } \\
\text { diagnosis, } n\end{array}$ & $\begin{array}{l}\text { Tumor } \\
\text { localization }\end{array}$ & $\begin{array}{l}\text { Treatment } \\
\text { regimen, } \mathrm{n}\end{array}$ & $\begin{array}{l}\text { Response } \\
\text { rates, } \mathrm{n}\end{array}$ & $\begin{array}{l}\text { Median PFS, } \\
\text { after start CT }\end{array}$ & $\begin{array}{l}\text { Median OS, } \\
\text { after start CT }\end{array}$ \\
\hline \multirow{4}{*}{$\begin{array}{l}\text { present } \\
\text { study }\end{array}$} & \multirow[t]{4}{*}{ Retrospective } & \multirow{4}{*}{$\begin{array}{l}\text { newly diagnosed } \\
\text { and recurrent } \\
\text { WHO grade II } \\
\text { and III } \\
\text { ependymoma, } 17\end{array}$} & \multirow{4}{*}{$\begin{array}{l}\text { supratentorial or } \\
\text { infratentorial }\end{array}$} & TMZ-based CT: 10 & CR: 1 & \multirow[t]{4}{*}{10 months } & \multirow[t]{4}{*}{41 months } \\
\hline & & & & platinum-based CT: 3 & PR: 1 & & \\
\hline & & & & PCV: 3 & SD: 11 & & \\
\hline & & & & $\begin{array}{l}\text { epirubicin/ifosfamide: } 1 \\
\text { (no prior } \mathrm{CT} \text { ) }\end{array}$ & PD: 4 & & \\
\hline \multirow{7}{*}{$\begin{array}{l}\text { Gilbert } \\
\text { et al., } \\
2014 \text { [16] }\end{array}$} & \multirow[t]{7}{*}{ Prospective } & \multirow{7}{*}{$\begin{array}{l}\text { recurrent WHO } \\
\text { grade II } \\
\text { ependymoma, } \\
24 \text { and grade III, } \\
18 \text { ependymoma }\end{array}$} & \multirow{7}{*}{$\begin{array}{l}\text { intracranial } \\
\text { and/or spinal }\end{array}$} & \multirow[t]{7}{*}{ TMZ plus lapatinib } & WHO grade II & WHO-grade II: 45 weeks & - \\
\hline & & & & & PR: 2 & \multirow{6}{*}{$\begin{array}{l}\text { WHO-grade III: } \\
25.3 \text { weeks }\end{array}$} & \\
\hline & & & & & $\begin{array}{l}\text { SD/PD: no } \\
\text { data }\end{array}$ & & \\
\hline & & & & & WHO grade III & & \\
\hline & & & & & CR: 1 & & \\
\hline & & & & & PR: 1 & & \\
\hline & & & & & $\begin{array}{l}\text { SD/PD: no } \\
\text { data }\end{array}$ & & \\
\hline \multirow{3}{*}{$\begin{array}{l}\text { Chamberlain } \\
\text { and Johnston, } \\
2009 \text { [15] }\end{array}$} & \multirow{3}{*}{ Retrospective } & \multirow{3}{*}{$\begin{array}{l}\text { recurrent WHO } \\
\text { grade II } \\
\text { ependymoma, } 25\end{array}$} & \multirow[t]{3}{*}{ supratentorial } & \multirow{3}{*}{$\begin{array}{l}\text { TMZ } \\
\text { (after platinum-based CT) }\end{array}$} & PR: 1 & \multirow[t]{3}{*}{2 months } & \multirow[t]{3}{*}{3 months } \\
\hline & & & & & SD: 9 & & \\
\hline & & & & & PD: 15 & & \\
\hline \multirow{3}{*}{$\begin{array}{l}\text { Green } \\
\text { et al., } \\
2009 \text { [26] }\end{array}$} & \multirow[t]{3}{*}{ Retrospective } & \multirow{3}{*}{$\begin{array}{l}\text { recurrent WHO } \\
\text { grade II and III } \\
\text { ependymoma, } 8\end{array}$} & \multirow{3}{*}{$\begin{array}{l}\text { supratentorial } \\
\text { or infratentorial }\end{array}$} & \multirow{3}{*}{$\begin{array}{l}\text { bevacizumab } \\
\text { (after platinum-based CT or TMZ) }\end{array}$} & PR: 6 & \multirow[t]{3}{*}{6.4 months } & \multirow[t]{3}{*}{9.4 months } \\
\hline & & & & & SD: 1 & & \\
\hline & & & & & PD: 1 & & \\
\hline \multirow{8}{*}{$\begin{array}{l}\text { Brandes } \\
\text { et al., } \\
2005 \text { [17] }\end{array}$} & \multirow[t]{8}{*}{ Retrospective } & \multirow{8}{*}{$\begin{array}{l}\text { recurrent WHO } \\
\text { grade II and III } \\
\text { ependymoma, } 28\end{array}$} & \multirow[t]{8}{*}{ intracranial } & \multirow{4}{*}{$\begin{array}{l}\text { cisplatin-based CT: } 13 \\
\text { (no prior CT) }\end{array}$} & CR: 2 & \multirow[t]{4}{*}{9.9 months } & 31 months \\
\hline & & & & & PR: 2 & & \\
\hline & & & & & SD: 7 & & \\
\hline & & & & & PD: 2 & & \\
\hline & & & & versus & versus & versus & versus \\
\hline & & & & CT without cisplatin: 15 & PR: 2 & 10.9 months & 40.7 months \\
\hline & & & & (no prior (1) & SD: 11 & & \\
\hline & & & & & PD: 2 & & \\
\hline
\end{tabular}


Table 4 Review of the literature: chemotherapeutic treatment regimens in adult intracranial ependymoma (Continued)

\begin{tabular}{|c|c|c|c|c|c|c|c|}
\hline \multirow{4}{*}{$\begin{array}{l}\text { Soffietti } \\
\text { et al., } \\
2005 \text { [14] }\end{array}$} & \multirow[t]{4}{*}{ Prospective } & \multirow{4}{*}{$\begin{array}{l}\text { recurrent WHO } \\
\text { grade II and III } \\
\text { ependymoma, } 11\end{array}$} & \multirow[t]{4}{*}{ intracranial } & \multirow{4}{*}{$\begin{array}{l}\text { TMZ } \\
\text { (some after nitrosourea or platinum-based CT) }\end{array}$} & CR: 2 & CR: 9-48+ months & \multirow[t]{4}{*}{-} \\
\hline & & & & & PR: 3 & PR: 4-15+ months & \\
\hline & & & & & SD: 5 & SD: $7-44+$ months & \\
\hline & & & & & PD: 1 & & \\
\hline $\begin{array}{l}\text { Lombardi } \\
\text { et al., } \\
2013 \text { [13] }\end{array}$ & case report & $\begin{array}{l}\text { recurrent anaplastic } \\
\text { ependymoma }\end{array}$ & supratentorial & $\begin{array}{l}\text { TMZ plus cisplatin } \\
\text { (after platinum-based CT alone and TMZ alone) }\end{array}$ & $\mathrm{PR}$ & 9 months & 11 months \\
\hline $\begin{array}{l}\text { Freyschlag } \\
\text { et al., } \\
2011 \text { [12] }\end{array}$ & case report & $\begin{array}{l}\text { recurrent anaplastic } \\
\text { ependymoma }\end{array}$ & supratentorial & TMZ & $\begin{array}{l}\text { no evidence of } \\
\text { radiographic } \\
\text { progression }\end{array}$ & 5+ months & - \\
\hline $\begin{array}{l}\text { Rojas-Marcos } \\
\text { et al., } \\
2003 \text { [25] }\end{array}$ & case report & $\begin{array}{l}\text { recurrent anaplastic } \\
\text { ependymoma }\end{array}$ & $\begin{array}{l}\text { infratentorial (initial) } \\
\text { and supratentorial } \\
\text { (at recurrence) }\end{array}$ & $\begin{array}{l}\text { tamoxifen plus isotretinoin } \\
\text { (after TMZ, platinum-based } C T, C C N U \text { ) }\end{array}$ & $C R$ & 17 months & - \\
\hline
\end{tabular}

$C R$ complete response, $C T$ chemotherapy, $n$ number of patients, $O S$ overall survival, $P C V$ procarbazine/lomustine/vincristine, $P D$ progressive disease, $P F S$ progression-free survival, $P R$ partial response, $S D$ stable disease, $T M Z$ temozolomide

+ indicates patients who did not demonstrate progressive disease 
lapatinib, were presented at the annual meeting of the Society of Neurooncology (SNO) in 2014 [16]. Lapatinib targets the epidermal growth factor receptor (ErbB1) and the related family member HER-2/neu (ErbB2) on the cell surface of the tumor cells. Median PFS was 45 weeks for patients diagnosed with grade II, and 25.3 weeks for patients diagnosed with grade III ependymomas. Best response rates were CR in one patient diagnosed with anaplastic astrocytoma and PR in 1 patient diagnosed with anaplastic astrocytoma and 2 patients diagnosed for ependymoma WHO grade II. Several patients showed at least SD. First results of a molecular classification revealed a correlation of response with ErbB2 expression [16]. In addition, there are two case reports of patients diagnosed with recurrent anaplastic ependymoma, describing a median PFS after TMZ treatment of 5 and 9 months [12,13] (Table 4). One reason for the possible failure of TMZ in patients with intracranial glioma is a non-methylated MGMT promoter [21]. Ependymomas may express high levels of MGMT [22], predicting less benefit from TMZ-based CT. Only 3 out of 12 ependymoma patients included in our study had tumors with a methylated MGMT promoter. Median PFS in these 3 patients was in the range of 1-6 months and thus low, and one of these patients treated with TMZ demonstrated no response. Our small dataset fails to indicate a favorable prognostic role of MGMT promoter methylation in adult ependymoma.

The other chemotherapeutic regimens were platinumbased CT $(n=3)$ or PCV $(n=3)$, showing a median PFS of 4 or 6 months and response rates as follows: PR (1 patient) or SD (3 patients) (Table 2). Brandes et al. published a retrospective study in 2005, analyzing 28 ependymoma patients (WHO grade II and III); 13 patients were treated with platinum-based $\mathrm{CT}$, demonstrating best responses of $2 \mathrm{CR}, 2 \mathrm{PR}$ and $7 \mathrm{SD}$, and a median PFS of 9.9 months in comparison to 15 patients treated with CT without cisplatin, demonstrating best responses of 2 PR and 11 SD, and a median PFS of 10.9 months [17]. PFS data were similar to the data reported here. Although response rates were lower in the group of patients treated with any other CT, but without cisplatin, survival curves (PFS and OS) did not differ. Three of the 15 patients were treated with $\mathrm{PCV}$, showing best responses with $1 \mathrm{PR}$, and $2 \mathrm{SD}$, therefore demonstrating a similar response as reported here (Tables 2 and 4).

Beyond these mentioned standard chemotherapeutic drugs, there is need for patient stratification based on molecular markers to identify ependymoma subgroups as well as subgroup-specific therapies, as recently described by Pajtler et al. [23]. Since all pre-clinical in vivo models tested for chemotherapy so far showed only reduced sensitivity towards these standard chemotherapeutic drugs, targeted therapies (e.g. epidermal growth factor receptor (EGFR) inhibitors, histone deacetylase (HDAC) inhibitors) are in the focus of research at the moment and need to be investigated [24].

Review of the literature showed a case report demonstrating a CR and a median PFS of 17 months in an adult patient with recurrent anaplastic intracranial ependymoma after treatment with tamoxifen and isotretinoin [25]. Beyond classical CT, Green et al. reported outcome data in 8 recurrent WHO grade II and III intracranial ependymomas which were treated with bevacizumab at time of recurrence. Promising response rates with 6 PR and 1 SD and a median PFS of 6.4 and OS of 9.4 months were described [26].

Two patients in our study were initially diagnosed with anaplastic ependymoma and at recurrence demonstrated sarcoma or gliosarcoma (Table 2). Both patients had been treated with RT in-between and had supratentorial tumors. A common origin for the initial and the recurrent tumor has to be considered, since there are reports in the literature demonstrating identical genetic mutations in both glial and sarcomatous compartments of gliosarcomas [16, 27, 28]. Interestingly, both patients appeared to derive benefit from chemotherapy.

Kaplan-Meier survival analysis revealed supratentorial tumor location as a parameter associated with inferior survival (Table 3). Supratentorial tumor localization has been described as significantly increasing risk of early death [29], therefore underlining the clinical aggressiveness of supratentorial ependymomas in adults that is independent from CT treatment. Our data suggest that survival outcomes in response to chemotherapy in adult intracranial ependymoma patients vary substantially, but individual patients may respond to any kind of chemotherapy.

\section{Conclusions}

The main limitation of our study is its retrospective design and the low number of patients in each chemotherapeutic subgroup. In summary, this retrospective study provides data supporting activity of TMZ in recurrent anaplastic ependymoma; however, there are also promising response rates in patients treated with platinumbased CT or PCV. Because of the notably individual survival outcomes after chemotherapeutic treatment in adult ependymoma patients with intracranial disease, prospective studies are urgently needed to identify patient subgroups that will benefit from individual chemotherapeutic treatments. Yet, this report suggests that at least one line of CT should be offered to ependymoma patients who are no longer candidates for surgery or RT.

\section{Availability of data and materials}

The dataset supporting the conclusions of this article is included within the article in Table 2. Full data on all 
patients are available in the database of the German Glioma Network (GGN) (http://www.gliomnetzwerk.de), Leipzig, Germany, that is not open for public. Data on patients from the University Hospital Zurich are available at a local database of the Department of Neurology, Zurich, Switzerland, that is also not open for the public.

\section{Additional file}

Additional file 1: Table S1 Association of age, tumor localization, gender, KPS and MGMT promoter methylation status with survival $\left(n=15\right.$, patients 5 and 9 excluded $\left.^{\circ}\right)$. (PDF $\left.82 \mathrm{~kb}\right)$

\section{Abbreviations}

ASCO: American Society of Clinical Oncology; Cl: confidence interval; CR: complete response; CT: chemotherapy; EGFR: epidermal growth factor receptor; GGN: German Glioma Network; HDAC: histone deacetylase; KPS: Karnofsky Performance Score; MGMT: $0^{6}$-methylguanyl-DNAmethyltransferase; OS: overall survival; PCV: procarbazine/lomustine/vincristine; PD: progressive disease; PFS: progression-free survival; PR: partial response; RT: radiotherapy; SD: stable disease; SNO: Society of Neurooncology; $\mathrm{TMZ}$ : temozolomide, TMZ/RT $\rightarrow \mathrm{TMZ}$, radiotherapy plus concomitant and maintenance temozolomide chemotherapy; WHO: World Health Organisation

\section{Competing interests}

PR has received honoraria for lectures or advisory boards from Roche, MSD, Molecular Partners, Novartis and Medac. SH has received a research grant from Roche. MaW has received honoraria for advisory board participations from Roche, BioMarin, PharmacoKinesis and Novocure. GR has received research grants from Roche and Merck as well as honoraria for advisory boards from Amgen, Celldex, Merck Serono and Roche. MiW has received research grants from Acceleron, Alpinia Institute, Bayer, Isarna, MSD, Merck Serono, Phytopharmaceutical Sciences and Roche and honoraria for lectures or advisory board participation from Celldex, Isarna, Magforce, MSD, Merck Serono, Pfizer, Roche and Teva. The other authors report no competing interests.

\section{Authors' contributions}

MiW and GR conceived this study. JF, EJR and GR contributed to the molecular analyses. DG carried out statistical analyses. DG, BH, PR, SH, MaW DK, MS, OS and WW contributed patient samples and clinical data. Manuscript was written by DG and MiW, with support from all authors. The final version of the article was reviewed and approved by all authors.

\section{Acknowledgements}

The authors would like to thank the staff at the clinical centers of the German Glioma Network, as well as the patients and their relatives.

\section{Funding}

This study was funded by the German Cancer Aid (grant no. 70-3163-Wi 3).

\section{Author details}

'Department of Neurology, University Hospital Zurich, Frauenklinikstrasse 26, 8091 Zurich, Switzerland. 'Department of Neuropathology, Heinrich-Heine-University, Moorenstrasse 5, 40225 Düsseldorf, Germany. ${ }^{3}$ German Cancer Consortium (DKTK), partner site Essen/Düsseldorf, German Cancer Research Center (DKFZ), Im Neuenheimer Feld 280, 69121 Heidelberg, Germany. ${ }^{4}$ Department of Oncology, University Hospital Zurich, Rämistrasse 100, 8091 Zurich, Switzerland. ${ }^{5}$ Department of Neuropathology, University Hospital Zurich, Schmelzbergstrasse 12, 8091 Zurich, Switzerland. ${ }^{6}$ Institute for Medical Informatics, Statistics and Epidemiology, University of Leipzig, Härtelstrasse 16-18, 04107 Leipzig, Germany. ${ }^{7}$ Department of Neurosurgery, University Medical Center Hamburg-Eppendorf, Martinistrasse 52, 20251 Hamburg, Germany. ${ }^{8}$ Department of Neurosurgery, Technical University Dresden, Fetscherstrasse 74, 01307 Dresden, Germany. ${ }^{9}$ Department of Neurosurgery, University of Bonn Medical School, Sigmund-Freud-Strasse 25, 53127 Bonn, Germany. ${ }^{10}$ Department of Neurosurgery, Ludwig Maximilian University Munich, Marchionistrasse 15 81377 Munich, Germany. ${ }^{11}$ Clinical Cooperation Unit Neurooncology, German
Cancer Consortium (DKTK), German Cancer Research Center (DKFZ), and Neurology Clinic and National Center for Tumor Diseases, University Hospital Heidelberg, Im Neuenheimer Feld 672, 69120 Heidelberg, Germany. ${ }^{12}$ Department of General Neurology, University Hospital Tübingen, Hoppe-Seyler-Strasse 3, 72076 Tübingen, Germany.

Received: 27 September 2015 Accepted: 19 April 2016

Published online: 23 April 2016

\section{References}

1. Ostrom QT, Gittleman H, Farah P, Ondracek A, Chen Y, Wolinsky Y, Stroup NE, Kruchko C, Barnholtz-Sloan JS. CBTRUS statistical report: primary brain and central nervous system tumors diagnosed in the United States in 2006-2010. Neuro Oncol. 2013;15 Suppl 2:ii1-56.

2. Gilbert MR, Ruda R, Soffietti R. Ependymomas in adults. Curr Neurol Neurosci Rep. 2010;10(3):240-7.

3. Vera-Bolanos E, Aldape K, Yuan Y, Wu J, Wani K, Necesito-Reyes MJ, Colman H, Dhall G, Lieberman FS, Metellus P et al. Clinical course and progressionfree survival of adult intracranial and spinal ependymoma patients. Neuro Oncol. 2015:17(3):440-7.

4. Louis DN, Ohgaki H, Wiestler B, Cavenee WK. WHO classification of tumours of the central nervous system. Lyon: IARC Press; 2007.

5. Guyotat J, Signorelli F, Desme S, Frappaz D, Madarassy G, Montange MF, Jouvet A, Bret P. Intracranial ependymomas in adult patients: analyses of prognostic factors. J Neurooncol. 2002;60(3):255-68.

6. Kawabata Y, Takahashi JA, Arakawa Y, Hashimoto N. Long-term outcome in patients harboring intracranial ependymoma. J Neurosurg. 2005;103(1):31-7.

7. Metellus P, Figarella-Branger D, Guyotat J, Barrie M, Giorgi R, Jouvet A, Chinot O. Club de Neuro-Oncologie de la Societe Francaise de N, the Association des Neuro-Oncologues d'Expression F. Supratentorial ependymomas: prognostic factors and outcome analysis in a retrospective series of 46 adult patients. Cancer. 2008;113(1):175-85.

8. Paulino AC, Wen BC, Buatti JM, Hussey DH, Zhen WK, Mayr NA, Menezes $\mathrm{AH}$. Intracranial ependymomas: an analysis of prognostic factors and patterns of failure. Am J Clin Oncol. 2002;25(2):117-22.

9. Merchant TE, Fouladi M. Ependymoma: new therapeutic approaches including radiation and chemotherapy. J Neurooncol. 2005;75(3):287-99.

10. Merchant TE, Li C, Xiong X, Kun LE, Boop FA, Sanford RA. Conformal radiotherapy after surgery for paediatric ependymoma: a prospective study. Lancet Oncol. 2009;10(3):258-66.

11. Reni M, Brandes AA, Vavassori V, Cavallo G, Casagrande F, Vastola F, Magli A Franzin A, Basso U, Villa E. A multicenter study of the prognosis and treatment of adult brain ependymal tumors. Cancer. 2004;100(6):1221-9.

12. Freyschlag CF, Tuettenberg J, Lohr F, Thome C, Schmieder K, Seiz M. Response to temozolomide in supratentorial multifocal recurrence of malignant ependymoma. Anticancer Res, 2011;31(3):1023-5.

13. Lombardi G, Pambuku A, Bellu L, Della Puppa A, Rumano L, Gardiman MP, Pomerri F, Zagonel V. Cisplatin and temozolomide combination in the treatment of supratentorial anaplastic ependymoma. Chemotherapy. 2013; 59(3):176-80.

14. Soffietti R, Costanza A, Laguzzi E, Trevisan E, Ruda R. Temozolomide in rare brain tumors of the adult: a prospective study. J Clin Oncol. 2005;23(16):132s.

15. Chamberlain MC, Johnston SK. Temozolomide for recurrent intracranial supratentorial platinum-refractory ependymoma. Cancer. 2009;115(20):4775-82.

16. Cachia D, Wani K, Penas-Prado M, Olar A, McCutcheon IE, Benjamin RS, Armstrong TS, Gilbert MR, Aldape KD. C11 orf95-RELA fusion present in a primary supratentorial ependymoma and recurrent sarcoma. Brain Tumor Pathol. 2015;32(2):105-11.

17. Brandes AA, Cavallo G, Reni M, Tosoni A, Nicolardi L, Scopece L, Franceschi E, Sotti G, Talacchi A, Turazzi S et al. A multicenter retrospective study of chemotherapy for recurrent intracranial ependymal tumors in adults by the Gruppo Italiano Cooperativo di Neuro-Oncologia. Cancer. 2005;104(1):143-8.

18. Felsberg J, Rapp M, Loeser S, Fimmers R, Stummer W, Goeppert M, Steiger HJ, Friedensdorf B, Reifenberger G, Sabel MC. Prognostic significance of molecular markers and extent of resection in primary glioblastoma patients. Clin Cancer Res. 2009:15(21):6683-93.

19. Macdonald DR, Cascino TL, Schold Jr SC, Cairncross JG. Response criteria for phase II studies of supratentorial malignant glioma. J Clin Oncol. 1990;8(7):1277-80.

20. Stupp R, Mason WP, van den Bent MJ, Weller M, Fisher B, Taphoorn MJ Belanger K, Brandes AA, Marosi C, Bogdahn U et al. Radiotherapy plus 
concomitant and adjuvant temozolomide for glioblastoma. N Engl J Med. 2005;352(10):987-96.

21. Weller M, Stupp R, Reifenberger G, Brandes AA, van den Bent MJ, Wick W, Hegi ME MGMT promoter methylation in malignant gliomas: ready for personalized medicine? Nat Rev Neurol. 2010;6(1):39-51.

22. Buccoliero AM, Castiglione F, Rossi Degl'Innocenti D, Paglierani M, Maio V, Gheri CF, Garbini F, Moncini D, Taddei A, Sardi I et al. O6-Methylguanine-DNAmethyltransferase in recurring anaplastic ependymomas: PCR and immunohistochemistry. J Chemother. 2008;20(2):263-8.

23. Pajtler KW, Witt H, Sill M, Jones DT, Hovestadt V, Kratochwil F, Wani K, Tatevossian R, Punchihewa C, Johann P et al. Molecular classification of ependymal tumors across all CNS compartments, histopathological grades, and age groups. Cancer Cell. 2015;27(5):728-43.

24. Witt H, Korshunov A, Pfister SM, Milde T. Molecular approaches to ependymoma: the next step(s). Curr Opin Neurol. 2012;25(6):745-50.

25. Rojas-Marcos I, Calvet D, Janoray P, Delattre JY. Response of recurrent anaplastic ependymoma to a combination of tamoxifen and isotretinoin. Neurology. 2003;61(7):1019-20.

26. Green RM, Cloughesy TF, Stupp R, DeAngelis LM, Woyshner EA, Ney DE, Lassman AB. Bevacizumab for recurrent ependymoma. Neurology. 2009;73(20):1677-80.

27. Actor B, Cobbers JM, Buschges $R$, Wolter $M$, Knobbe CB, Lichter $P$, Reifenberger $\mathrm{G}$, Weber RG. Comprehensive analysis of genomic alterations in gliosarcoma and its two tissue components. Genes Chromosomes Cancer. 2002;34(4):416-27.

28. Reis RM, Konu-Lebleblicioglu D, Lopes JM, Kleihues P, Ohgaki H. Genetic profile of gliosarcomas. Am J Pathol. 2000;156(2):425-32.

29. Sayegh ET, Aranda D, Kim JM, Oh T, Parsa AT, Oh MC. Prognosis by tumor location in adults with intracranial ependymomas. J Clin Neurosci. 2014; 21(12):2096-101.

\section{Submit your next manuscript to BioMed Central and we will help you at every step:}

- We accept pre-submission inquiries

- Our selector tool helps you to find the most relevant journal

- We provide round the clock customer support

- Convenient online submission

- Thorough peer review

- Inclusion in PubMed and all major indexing services

- Maximum visibility for your research

Submit your manuscript at www.biomedcentral.com/submit 